\title{
Immunosuppression and herpes viral reactivation in intensive care unit patients: one size does not fit all
}

\author{
Julien Textoris ${ }^{1,2^{*}}$ (D) and François Mallet ${ }^{1}$ \\ See related research by Mirouse et al., https://ccforum.biomedcentral.com/articles/10.1186/s13054-017-1731-0
}

Keywords: Viral reactivation, Herpes, Immunosuppression

More than 20 years after the initial description of cytomegalovirus (CMV) pneumonia in "non-immunocompromised" ICU patients by Papazian et al. [1], the treatment of herpes viruse reactivation in ICU is still a matter of debate. Recently, Mirouse et al. [2] reported a unique cohort of varicella-zoster virus (VZV)-related community acquired pneumonia in the ICU over 20 years. This highlighted that VZV infections in ICU patients are rare, with roughly a hundred cases over 20 years, in 29 French ICUs. Conversely, addressing a wider range of herpes viruses, two major papers recently reported a high frequency of viral reactivation in critically ill patients, and both showed variable plasma levels of various herpes viruses (Table 1) [3, 4]. Interestingly, almost $30 \%$ of the patients had multiple viremia events, and this viremia usually lasted until ICU discharge. The timing of viral reactivation was also informative, with herpes simplex virus (HSV)1 and Epstein-Barr virus (EBV) being detected earlier in the ICU course than CMV or human herpesvirus (HHV)6.

Several herpes viruses have been associated repeatedly with mortality and the occurrence of secondary infections. The most validated association is between CMV and mortality, either in ICU or at various times postICU [3-5]. In the MARS cohort, this association remained significant after adjusting for confounders, time-dependent bias, and competing risks [4]. Importantly, this association between CMV viremia and ICU

\footnotetext{
* Correspondence: julien.textoris@biomerieux.com

"EA7426 "Pathophysiology of Injury-Induced immunosuppression", Hospices

Civils de Lyon-Université Claude Bernard Lyon 1-bioMérieux, Lyon, France

${ }^{2}$ Anesthesiology and Critical Care Medicine, Hospices Civils de

Lyon-Université Claude Bernard Lyon 1, Lyon, France
}

mortality was significant (adjusted sdHR $=3.2[1.4-7.1]$ ) while taking into account other viral reactivation, which was never considered before [4]. Multiple reactivations might also suggest increased severity since the association of HHV6 and CMV reactivation was also associated with an increased risk of death in critically ill patients [6]. Association between EBV reactivation and mortality was also described [4,7]. Data are less clear for other neurotropic herpes viruses such as HSV2 or $\mathrm{VZV}$, which exhibit lower reactivation rates. Finally, in septic patients, CMV/EBV and HSV1 have also been associated with an increased rate of secondary fungal and bacterial infections, respectively [4]. This last result supports the hypothesis that immunosuppression might play an important role in this reactivation.

Most data related to critically ill patients made a clear distinction between immunocompromised (mainly oncologic treated patients, solid or bone marrow transplants) and so-called immunocompetent patients. However, we know that 30 to $50 \%$ of these critically ill patients exhibit signs of immunosuppression [8]. Clearly, the wording immunocompetent-to identify a category of patients that do not exhibit a severe iatrogenic or congenital immunosuppression-is misleading and it is time to propose several levels of immunosuppression (in terms of depth or type of immunosuppression) to better reflect the various levels of depressed immune status in ICU patients. Herpes viremia might therefore reflect either a lack of a latent virus control or a true clinical viral infection.

The ability to describe several types or levels of immunosuppression is important and supports a wider use of quantitative tools to measure viral titers in addition to host response biomarkers of the immune 
Table 1 Cumulative percentages of reactivation of various herpes viruses in septic patients during ICU stay

\begin{tabular}{lll}
\hline Virus & Walton et al. [3] & Ong et al. [4] \\
\hline VZV & - & $0.6 \%$ \\
HSV1 & $14 \%^{a}$ & $26 \%\left(31 \%^{\mathrm{a}}\right)$ \\
HSV2 & - & $4 \%$ \\
HHV6 & $10 \%$ & $24 \%$ \\
CMV & $24 \%^{\mathrm{a}}$ & $18 \%\left(27 \%^{\mathrm{a}}\right)$ \\
EBV & $53 \%$ & $48 \%$ \\
\hline
\end{tabular}

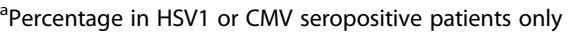

status. Considering the fact that many bone marrow transplant patients reactivate VZV [9] whereas VZV reactivation is almost absent in critically ill patients (this is supported also by the paucity of VZV severe pneumonia cases reported by Mirouse et al. over 20 years [2]), monitoring a panel of herpes viruses might also provide information about the type/depth of immunosuppression, and should be explored in more details. More generally, a quantitative and qualitative altered state of the plasma virome was observed in tacrolimus [10] or HIV-1 [11] induced immunosuppression, notably highlighting the correlation of the anellovirus viral load with the extent of immunosuppression.

Quantitative tools should allow us to define several thresholds to discriminate between (1) non-significant viral load, (2) viral "reactivation" as a marker of immunosuppression, and (3) high viral loads supporting a true viral infection requiring treatment. Such stratification with various levels of viremia might also be key to stratify patients for trials assessing the effectiveness of prophylactic/pre-emptive/or curative antiviral treatment in ICU patients [12]. It might also be part of the stratification tools/criteria used to select appropriate immunotherapy to critically ill patients $[13,14]$. It is important to consider that the global virome (including endogenous retroviruses), as part of the collective microbiome (and in addition to intrinsic and environmental factors), shape and regulate our immune system [15]. As commensal viruses might benefit the host [16], we should carefully consider the administration of antivirals, which will profoundly manipulate our virome and may end up doing more harm than good. Knowledge and tools are needed to better assess the host response, guide therapeutics, and avoid disruption in virome/microbiome-host homeostasis.

\section{Acknowledgements}

Not applicable.

Funding

Not applicable.

Availability of data and materials Not applicable.
Authors' contributions

JT and FM wrote and reviewed the manuscript. Both authors read and approved the final manuscript.

Ethics approval and consent to participate

Not applicable.

Consent for publication

Not applicable.

Competing interests

JT and FM are employee of bioMérieux. JT is an employee of Hospices Civils de Lyon.

\section{Publisher's Note}

Springer Nature remains neutral with regard to jurisdictional claims in published maps and institutional affiliations.

Published online: 26 August 2017

\section{References}

1. Papazian L, Fraisse A, Garbe L, Zandotti C, Thomas P, Saux P, et al. Cytomegalovirus an unexpected cause of ventilator-associated pneumonia. Anesthesiology. 1996;84:280-7.

2. Mirouse A, Vignon P, Piron P, Robert R, Papazian L, Géri G, et al. Severe varicella-zoster virus pneumonia: a multicenter cohort study. Crit Care. 2017; 21:137.

3. Walton AH, Muenzer JT, Rasche D, Boomer JS, Sato B, Brownstein BH, et al. Reactivation of multiple viruses in patients with sepsis. PLoS One. 2014;9: e98819.

4. Ong DSY, Bonten MJM, Spitoni C, Verduyn Lunel FM, Frencken JF, Horn J, et al. Epidemiology of multiple herpes viremia in previously immunocompetent patients with septic shock. Clin Infect Dis. 2017:64:1204-10.

5. Kalil AC, Florescu DF. Is cytomegalovirus reactivation increasing the mortality of patients with severe sepsis? Crit Care. 2011;15:138.

6. Lopez Roa P, Perez-Granda MJ, Munoz P, Catalan P, Alonso R, Sanchez-Perez $E$, et al. A prospective monitoring study of cytomegalovirus infection in non-immunosuppressed critical heart surgery patients. PLoS One. 2015;10: e0129447.

7. Libert N, Bigaillon C, Chargari C, Bensalah M, Muller V, Merat S, et al. Epstein-Barr virus reactivation in critically ill immunocompetent patients. Biomed J. 2015:38:70-6.

8. van der Poll T, van de Veerdonk FL, Scicluna BP, Netea MG. The immunopathology of sepsis and potential therapeutic targets. Nat Rev Immunol. 2017;17(7):407-20.

9. Vermont $\mathrm{CL}$, Jol-van der Zijde ECM, Hissink Muller P, Ball LM, Bredius RGM, Vossen AC, et al. Varicella zoster reactivation after hematopoietic stem cell transplant in children is strongly correlated with leukemia treatment and suppression of host T-lymphocyte immunity. Transpl Infect Dis. 2014;16:188-94.

10. De Vlaminck I, Khush KK, Strehl C, Kohli B, Luikart H, Neff NF, et al. Temporal response of the human virome to immunosuppression and antiviral therapy. Cell. 2013;155:1178-87.

11. Li L, Deng $X$, Linsuwanon P, Bangsberg D, Bwana MB, Hunt $P$, et al. AIDS alters the commensal plasma virome. J Virol. 2013;87:10912-5.

12. Cowley NJ, Owen A, Shiels SC, Millar J, Woolley R, Ives N, et al. Safety and efficacy of antiviral therapy for prevention of cytomegalovirus reactivation in immunocompetent critically ill patients: a randomized clinical trial. JAMA Intern Med. 2017;177:774-83.

13. van der Poll T, Opal SM. Host-pathogen interactions in sepsis. Lancet Infect Dis. 2008;8:32-43.

14. Rol M-L, Venet F, Rimmele T, Moucadel V, Cortez P, Quemeneur L, et al. The REAnimation Low Immune Status Markers (REALISM) project: a protocol for broad characterisation and follow-up of injury-induced immunosuppression in intensive care unit (ICU) critically ill patients. BMJ Open. 2017;7:e015734.

15. Mavrommatis B, Young GR, Kassiotis G. Counterpoise between the microbiome, host immune activation and pathology. Curr Opin Immunol. 2013;25:456-62.

16. Kernbauer E, Ding Y, Cadwell K. An enteric virus can replace the beneficial function of commensal bacteria. Nature. 2014;516:94-8. 